\title{
Distúrbio de Percepção Temporal e sua Influência na Memória: Estudo de Caso de Paciente com Lesão Frontal
}

\author{
Maria Alice de Mattos Pimenta Parente ${ }^{12}$ \\ Mônica Sparta \\ Universidade Federal do Rio Grande do Sul \\ André Luiz Palmini ${ }^{3}$ \\ Pontifícia Universidade Católica do Rio Grande do Sul
}

\begin{abstract}
Resumo
Os mecanismos da memória episódica possibilitam uma pessoa "viajar" pelo tempo, e quando relacionados ao tempo futuro, compõem a memória prospectiva. O objetivo desse trabalho é verificar, através de um estudo de caso de uma paciente com lesão cerebral no lobo frontal, apresentando distúrbio de percepção temporal e falhas de memória, se é possível diferenciar os mecanismos neurocognitivos de memória operacional dos da memória prospectiva. Tomando por base o modelo de Ellis, a paciente foi submetida a uma bateria de lobo frontal e à prova experimental proposta por Einstein e Daniel. Os resultados mostraram que suas falhas não afetam recursos atencionais necessários na execução de ações complexas, mas o controle de mecanismos internos (self-driven), como a noção do tempo, ativação de intenções e tomada de decisões. De acordo com o modelo adotado, a paciente apresenta falha específica na ativação de intenções e no processo de supervisão. Esses achados indicam que memória operacional e memória prospectiva podem ter correlatos neuropsicológicos diversos.

Palavras-chave: Memória prospectiva; memória operacional; neuropsicologia; lobo frontal.
\end{abstract}

Temporal Perception Disorder and its Influence on Memory:

A Case Study of a Patient with Frontal Lobe Lesion

\begin{abstract}
The cognitive mechanisms of the episodic memory allow a person to travel through time. These mechanisms that are related to the future compose the so-called prospective memory. The main objective of this work was to verify whether it is possible to dissociate neurocognitive mechanisms of the working memory from those of the prospective memory by using a case study with frontal lesion, presenting failures in time perception and memory disorders. Based on the Ellis model, the patient was tested using both a frontal lobe battery and an Einstein and Daniel's task. The results showed that the failures did not affect attentional resources required for the execution of complex actions, but decreased the self-driven mechanisms such as the notion of time, intentionality and decision making. Following the model, the patient had specific failures in the activation of intentions and in the supervision process. These failures suggest that both the working memory and the prospective memory may have different neuropsychological attributes.

Keywords: Prospective memory; working memory; neuropsychology; frontal lobe.
\end{abstract}

A habilidade de "viajar mentalmente pelo tempo possibilita uma pessoa reviver experiências retomando situações que ocorreram no passado e projetá-las mentalmente antecipando o futuro através da imaginação." (Wheeler, Stuss \& Tulving, 1997, p. 331).

\footnotetext{
${ }^{1}$ Endereço para correspondência: Universidade Federal do Rio Grande do Sul, Instituto de Psicologia Rua Ramiro Barcelos, 2600, sala 112, 90035 003, Porto Alegre, RS. Fone: (51) 33165111, Fax: (51) 33404797. E-mail: malicemp@zaz.com.br.

${ }^{2}$ Este estudo insere-se no âmbito de pesquisa sobre neuropsicologia do envelhecimento, subvencionada pelo CNPq e com apoio do convênio CAPES/SCyT.

${ }^{3}$ Faculdade de Medicina
}

Esta ponte entre experiências passadas e previsões do futuro é um dos mais importantes mecanismos de memória. Para Ades (1993) a memória "é, basicamente, esta intrusão do passado no presente, seja sob a forma de imagens, seja como instruções implícitas ou explícitas de como agir" (p. 9). Xavier (1993) considera-a uma "capacidade de alterar o comportamento em função de experiências anteriores” (p. 62). Xavier (1999) em comunicação pessoal, relatou que a memória é um processo básico para a sobrevivência, com a finalidade primordial de gerar previsões. A memória denominada episódica fornece esses mecanismos cognitivos que possibilitam a viagem pelo tempo (Wheeler e cols., 1997). Através dela temos lembranças subjetivas de nossas 
experiências, somos capazes de voltar ao tempo e ao contexto em que vivemos, o que contrasta com a armazenagem objetiva e descontextualizada da memória chamada semântica (Van der Linden, 1994; Xavier, 1993). Ambas memórias, episódica e semântica, têm sido consideradas memórias de longo prazo declarativas que associam ao presente as informações que ocorreram no passado, sendo chamadas aqui, memórias retrógradas (Wiggs, Weisberg \& Martin, 1999).

Recentemente, estudos da Psicologia Cognitiva e da Neuropsicologia têm focalizado memórias que relacionam tempos presentes a futuros, as chamadas memórias prospectivas (Brandimonte, Einstein \& McDaniel, 1996). Ao programar uma atividade numa data futura, uma pessoa ativa mecanismos especiais de memória que serão acionados num tempo préestabelecido. Para Wheeler e colaboradores (1997), o conteúdo subjetivo e intencional é a principal característica da memória prospectiva.

Dificuldades em organizar as atividades diárias têm sido descritas em pacientes portadores de lesões frontais adquiridas após traumatismos, acidentes vasculares ou tumores (Mayes \& Daum, 1997; Stuss \& Benson, 1986). Estes pacientes mantém preservadas suas funções motoras, lingüísticas e várias formas de raciocínio. Entretanto, apresentam grandes problemas de adaptação social e de organização de suas atividades e, em alguns casos, apresentam alterações evidentes de controle emocional. Surgem problemas familiares, não se mantém no emprego e são incapazes de organizar sua própria vida. A variabilidade de manifestações desses pacientes pode decorrer dos diferentes mecanismos cognitivos que têm suportes de distintas áreas do córtex pré-frontal (Fuster, 1999; Luria, 1966). Alguns pacientes apresentam desempenho adequado em muitas provas neuropsicológicas, o que dificulta o diagnóstico, mas também estimula o estudo da natureza básica dessas dificuldades (Damásio, 1996; Gainotti, 1994).

Para Burgess e Shallice (1997), os pacientes com lesão frontal têm muitas dificuldades de organizar suas atividades, o que acontece nas situações em que os comportamentos são guiados por intenções explícitas, geradas anteriormente; ou quando existe necessidade de atuar conforme regras pré-estabelecidas; ou ainda quando é preciso tomar decisões elaboradas em um tempo anterior. Os autores dizem existir "marcadores de natureza temporal" originados no estabelecimento de intenções ou quando regras são temporariamente criadas. Tais intenções ou regras de comportamento serão, por sua vez, ativadas na presença de uma situação relevante e em determinado tempo. Em outras palavras, para esses autores, existe um espaço de latência entre a criação dos

Tabela 1. Resultados da Bateria de Lobo Frontal

\begin{tabular}{|c|c|}
\hline Área/Provas & Desempenho \\
\hline \multicolumn{2}{|l|}{ Raciocínio } \\
\hline Abstração de informações e de propriedades & adequado \\
\hline Formulação de estratégias estimativas: & adequado \\
\hline Resolução de problemas aritméticos & adequado \\
\hline T. Hanoi -Processos antecipatórios & dificuldade \\
\hline Wisconsin - flexibilidade e percepção de estratégia do outro & dificuldade \\
\hline \multicolumn{2}{|l|}{ Atenção } \\
\hline cancelamento de Brickenkamp & adequado \\
\hline Trail Making & adequado* \\
\hline Teste de Stroop & interferência maior* \\
\hline \multicolumn{2}{|l|}{ Memória } \\
\hline Span de Dígitos: & adequado** \\
\hline Span de Palavras & adequado** \\
\hline Prova de aprendizagem verbal de Rey & retenção superior*/perseverações \\
\hline Memória textual & adequado*** $^{* *}$ \\
\hline Memória operacional com interferência & adequado \\
\hline Tarefas ecológicas de memória prospectiva & falho \\
\hline
\end{tabular}

* conforme normas de Spreen e Strauss (1991)

** conforme normas de Chaves e Izquerdo (1992)

*** conforme normas de Parente, Capuano e Nespoulous (1999) 
marcadores temporais e sua ativação. Pacientes com lesão pré-frontal perdem a capacidade de ativar tais marcadores temporais e a capacidade de organizar a ordem temporal de elementos verbais ou visuais aprendidos (Milner, Corst \& Leonard, 1991).

Grande parte das ações do cotidiano é realizada espontaneamente sem necessidade de formulação prévia. Nestas, mesmo sendo ações intencionais, a simultaneidade de ação/intenção impede a distinção da última. Entretanto, outras ações são planejadas para ocorrer após um intervalo de tempo. Searle (1983) acredita que, inicialmente, é formada uma intenção e uma ação. A intenção desloca o seu objeto - ou seja, a ação - para um determinado tempo no futuro. Quando chegar esse tempo, a intenção deve ser ativada por pistas, intrínsecas ou extrínsecas ao sujeito, e recuperada do passado. Nesta perspectiva, uma característica que diferencia a tarefa de memória prospectiva da retrospectiva é que a primeira exige geração própria (self-initiation) enquanto a segunda é eliciada pelo examinador (Einstein \& Daniel, 1990).

A memória prospectiva, segundo Ellis (1996), pode ser dividida em quatro fases (Figura 1): (1) codificação e elaboração de planos de ações que incluem as intenções de sua realização e um contexto imaginário de recuperação destas intenções; (2) intervalo de tempo, onde diferentes eventos podem auxiliar a evocação da intenção de realizar uma ação; (3) realização da ação planejada; e, (4) avaliação dos resultados dessa ação, com um sistema de supervisão do resultado da ação. O ponto crucial da memória prospectiva parece ser sua natureza intencional e não o planejamento da ação e os processos de recuperação. Neste sentido, uma intenção é uma ação a ser realizada em um tempo específico do futuro. Para Ellis (1996), somente quando se abre um espaço entre o presente e uma ação futura concreta é que está se exigindo uma memória prospectiva. Por esta razão, este sistema de memória tem recebido diferentes nomes: memória prospectiva (Dalla Barba, 1993), memória intencional (Goschke \& Kuhl, 1996; Kvavilashvili, 1987); memória

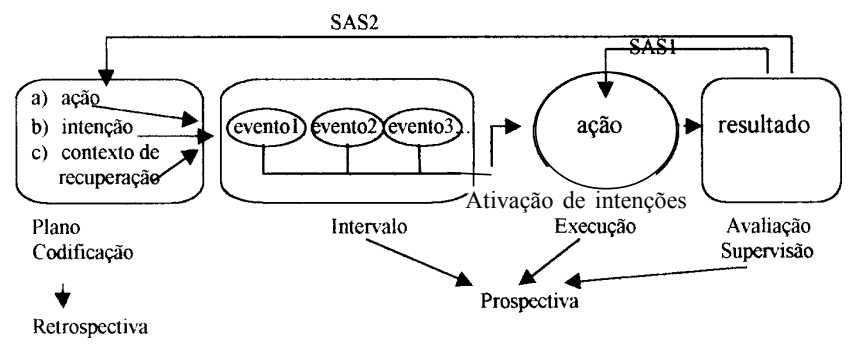

Figura 1. Fases da memória prospectiva (Ellis, 1996) para atividades diárias (Cohen, 1991) e memória do futuro (Einstein \& Daniel, 1990).

A questão do espaço de tempo entre a intenção e a execução, nem sempre é clara nos trabalhos neuropsicológicos, o que torna confusa a nomenclatura dos diferentes tipos de memória. Cohen (1991) utilizou a designação memória prospectiva para planos de ações imediatas e retardadas. Por outro lado, em nosso meio, Xavier (1993, p.107; 1996) denominou memória operacional um tipo de memória transitória que pode manter informações por períodos variáveis de tempo em função de sua utilidade. Por enfatizar apenas seu produto e não os processos psicológicos envolvidos, sua definição engloba tanto o que aqui chamamos de memória prospectiva, assim como o que denominamos memória operacional (working memory, no sentido de Baddeley, 1986), cujo componente primordial são seus recursos atencionais.

Segundo Richardson (1996), Miller, Galanter e Pribam foram os primeiros a utilizar, em 1960, o termo "memória operacional" (working memory) considerando o lobo frontal como responsável pela 'memória operacional', na qual os planos podem ser retidos temporariamente quando estão sendo formados, transformados ou executados. Neste sentido é um sistema de processamento da informação que atua no controle executivo da cognição e do comportamento, sendo um tipo de memória de curto prazo. Esta interpretação, apesar de genérica, é bastante aceita pela neuropsicologia atual.

O conceito de memória operacional não foi muito valorizado no modelo de Atkinson e Shiffrin (1971), que propunha três sistemas de memória distintos: sensorial, memória de curto prazo e memória de longo prazo. Mesmo assim, os autores afirmam que a "memória de curto prazo pode ser uma memória operacional, onde as decisões são realizadas, os problemas resolvidos e o fluxo de informação direcionado" (p. 83).

Baddeley e Hitch (1974) iniciaram uma série de pesquisas desenvolvendo o conceito de memória operacional (working memory). Ela é capaz de manter ativadas diferentes informações pelo tempo necessário para a execução de uma tarefa complexa. Seu elemento principal, o "executivo central", possui recursos atencionais que possibilitam a execução de tarefas concomitantes, necessárias em diferentes situações-problema, como resolução de problemas matemáticos, compreensão de leitura textual, etc. Este executivo central mantém contato com as memórias de longo prazo e coordena o trabalho de processos fonológicos e/ou visuo-espaciais.

Denominaremos memória operacional (working memory), nesse estudo, aquela que coordena atividades que 
estão sendo realizadas numa atividade complexa e que requer primordialmente recursos atencionais. $\mathrm{O}$ termo memória prospectiva será utilizado para as tarefas projetadas em um futuro, ou seja, com um tempo entre intenção e ação e, conseqüentemente, exigindo a ativação de intenções planejadas anteriormente num momento exato.

Surge então uma questão: existe um sistema específico de memória prospectiva? Burgess e Shallice (1997), ao estudarem três pacientes com lesão frontal, observaram que aqueles que possuíam dificuldades de memória retrospectiva (episódica) também possuíam dificuldades em memória prospectiva; entretanto, alguns pacientes só possuíam dificuldades em memória prospectiva. A ausência de uma dupla dissociação, segundo esses autores, parece indicar que a memória prospectiva é composta de alguns componentes da retrospectiva além de outros que lhe são próprios. Contrariamente, outros trabalhos sugerem a independência deste tipo de memória. Kvavilasvili (1987) não encontrou correlação entre o lembrar uma intenção e o lembrar o conteúdo em um estudo experimental enquanto que Wilkins e Baddeley (1978) encontraram correlação negativa em tarefas naturalísticas.

Neste trabalho, através de um estudo de caso de uma paciente com lesão cerebral no lobo frontal, estudaremos as razões de sua queixa de incapacidade de organizar atividades, do relato de perda da noção subjetiva da percepção de tempo e de falhas na memória, tomando como base modelos cognitivos de memória prospectiva. Também procuraremos apontar uma dissociação entre mecanismos atencionais e intencionais de diferentes tipos de memórias (memória operacional e prospectiva) que atuam nas atividades da vida cotidiana. Reconhecendo que a dificuldade em memória prospectiva ocorre por falhas na memória episódica em função de sua natureza temporal, levantamos a questão se ela pode ser diferenciada da memória operacional (working memory), a partir de uma distinção entre os processos atencionais e intencionais.

\section{Método}

\section{Dados Pessoais}

A paciente $A B$, do sexo feminino, tinha 38 anos na época da avaliação. Brasileira, fez curso universitário no Brasil e mestrado nos Estados Unidos, onde casou e ficou residindo. Em agosto de 1996, sofreu um acidente de carro. Ao voltar para casa, dormiu muito e pareceu desorientada no tempo, durante um período de três dias. Ao perceber sangramento na orelha, foi novamente internada, recebendo logo alta. Foi liberada de seu trabalho e não conseguia organizar-se em casa. Quando resolvia arrumar um quarto, por exemplo, podia passar horas sem sentir o tempo passar. Não lembrava onde havia guardado suas coisas, o que lhe deu muitos problemas no condomínio, devido às sucessivas perdas do cartão para abrir a porta da garagem. Seu marido perguntava-lhe com freqüência o que tinha feito durante o dia e ela não se lembrava; quando ele queria saber por que ela guardara determinado objeto em um lugar, ela igualmente não sabia o que responder.

$\mathrm{AB}$ relatou que apesar de, normalmente, sentir emoções, um dia viu uma cena impressionante num filme, mas não sentira qualquer emoção e achou muito estranha sua falta de espanto. Procurou, por muito tempo, uma resposta, até que, semanas depois, com muito esforço conseguiu lembrar que já havia visto a foto daquela cena numa revista sobre cinemas. Uma grande dificuldade para $\mathrm{AB}$ é fazer compras no supermercado. Mesmo que leve uma lista para se lembrar do que precisa, $\mathrm{AB}$ fica muito tempo olhando para os diversos produtos e demora bastante até se decidir por um deles. A relação entre o casal foi piorando e a mãe de $\mathrm{AB}$ resolveu trazê-la ao Brasil, onde foi submetida a uma avaliação neurológica e neuropsicológica, três anos depois do acidente. A paciente foi submetida à ressonância magnética nuclear e ao SPECT que apontaram lesão na região pré-frontal.

\section{Material}

Triagem Inicial

Inicialmente $\mathrm{AB}$ foi submetida a uma entrevista aberta, onde observou-se sua linguagem e sua memória autobiográfica. Também foi incluído nessa triagem o teste Mini-mental (adaptação de Chaves \& Izquierdo, 1992), utilizado para detecção inicial de distúrbios degenerativos. Ele consta de seis tarefas que verificam: (1) capacidade de orientar no espaço e no tempo; (2) retenção de três palavras; (3) capacidades de cálculos aritméticos e atencionais; (4) memória recente; (5) habilidades lingüísticas; e, (6) praxis construtivas.

\section{Provas para Investigação de Funções de Lobo Frontal \\ Provas de Raciocínio}

(1) Abstração de Informações e de Propriedades: Foi solicitada a $\mathrm{AB}$ a interpretação de três provérbios ("quem com ferro fere, com ferro será ferido"; "quem não tem cão, caça com gato" e "água mole em pedra dura, tanto bate até que fura"); a descrição das diferenças entre palavras (anão/criança e roubo/engano) e definições de palavras.

Psicologia: Reflexão e Crítica, 2001, 14(2), pp. 343-352 
(2) Formulação de Estratégias Estimativas: Foram apresentados a $\mathrm{AB}$ problemas que verificam capacidades de se estimar valores como, por exemplo: (1) se um sapato custasse 400 reais, qual seria o preço do quilo de carne e de uma casa? e, calcular a velocidade média do galope de cavalos; a medida média da coluna vertebral dos homens; e a altura de uma casa de dois andares.

(3) Processos Antecipatórios e Organização de Seqüências de Ações: Foi solicitado a $\mathrm{AB}$ realizar o problema da Torre de Hanói, com cinco círculos, numa versão computadorizada.

(4) Avaliação da Estratégia do Interlocutor e da Capacidade de Flexibilidade Mental: Foi apresentada a AB uma versão computadorizada do teste de Wisconsin (Spreen \& Strauss, 1991). Neste teste, são apresentadas cartas que devem ser associadas a uma entre quatro cartas, de acordo com um critério não conhecido pelo paciente, que pode ser cor, forma ou número. O computador apenas assinala se a escolha foi "certa" ou "errada". Quando o paciente acerta um critério por 10 vezes consecutivas, este é alterado, de forma que a resposta considerada "correta" passa a ser "errada".

\section{Provas de Atenção}

(1) Prova de Cancelamento de Brickenkamp (Spreen \& Strauss, 1991): Este teste verifica a manutenção da atenção. Foi apresentado à $\mathrm{AB}$, após um treino prévio, uma folha com 14 seqüências de letras d e p, ordenadas aleatoriamente. $\mathrm{AB}$ foi instruída a riscar somente as letras "d" e, após 1 minuto, o examinador solicitava que mudasse de linha, continuando o cancelamento.

(2) Trail Making (Spreen \& Strauss, 1991): Este teste, além de manutenção da atenção, verifica, na terceira prova, a capacidade de alternância entre um critério e outro. São três provas, nas quais o paciente deve formar um caminho ligando letras e/ou números. A primeira folha apresenta números que devem ser ligados em ordem crescente; a segunda, letras que devem ser ligadas em ordem alfabética; e a terceira, letras e números que devem ser ligados de forma intercalada, na ordem crescente para os números, e alfabética para as letras, com registro de tempo.

(3) Teste de Stroop: Este teste verifica rapidez e capacidade em suprimir respostas habituais e bastante automáticas, em favor de não usuais. Foi utilizada a versão de Vitória, apresentada em Spreen e Strauss (1991), consistindo de três cartões de 21,5 por $14 \mathrm{~cm}$. cada um, contendo seis colunas de quatro elementos. No primeiro cartão, o sujeito deve nomear, o mais rapidamente possível, a cor de 24 pequenos retângulos apresentados em rosa, verde azul e marrom. No segundo cartão, o sujeito deve nomear as cores apresentadas em palavras

Psicologia: Reflexão e Crítica, 2001, 14(2), pp. 343-352 que designam preposições ou advérbios, ou seja, palavras de pouco conteúdo semântico. No último, com a mesma tarefa, as palavras designam nomes de cores diferentes das cores de suas letras.

\section{Provas de Memória}

(1) Span de Dígitos: Foram apresentadas séries de dígitos para repetição, contendo duas séries com o mesmo número de dígitos, do Protocolo de Positividade para déficit cognitivo (Chaves \& Izquierdo, 1992).

(2) Span de Palavras: Foi solicitada a repetição de dez palavras freqüentes que também se encontra no Protocolo de Positividade para déficit cognitivo (Chaves \& Izquierdo, 1992).

(3) Prova de Aprendizagem Verbal de Rey: Uma adaptação realizada da prova de Rey consistiu em cinco apresentações de 16 palavras de quatro categorias (partes do corpo, animais, membros da família e objetos da casa). $\mathrm{AB}$ foi informada que ninguém consegue lembrar todas as palavras de uma só vez. Por esta razão, as palavras seriam repetidas cinco vezes. Após, foi apresentada outra lista e solicitada a repetição da anterior. Por fim, após 30 minutos, $\mathrm{AB}$ repetiu a seqüência inicial.

(4) Memória Textual: Foi relatada oralmente a história de velho homem (Cadillac, Virbel \& Nespoulous, 1995; Parente, Capuano \& Nespoulous, 1999) e solicitado seu reconto.

(5) Memória Operacional com Interferência: Foi solicitada a repetição de três letras após 10 e 20 segundos, concomitante ao movimento de dedilhar sobre a mesa.

(6) Tarefas Ecológicas de Memória Prospectiva: Duas tarefas de memória prospectiva, utilizando atividades simples, foram solicitadas à AB: (1) lembrar o examinador para telefonar ao seu dentista ao terminar a sessão e (2) no dia seguinte, telefonar-lhe às oito horas da noite.

(7) Prova Experimental de Memória Prospectiva e Memória de Trabalho: Foi realizada uma adaptação da prova de Einstein e Daniel (1990). No início da sessão, $\mathrm{AB}$ foi avisada de que iria fazer várias provas de memória. A última delas, que seria realizada no final da sessão, visava verificar a capacidade de retenção da memória de curto prazo. Seriam apresentadas 35 seqüências de algarismos para serem repetidas logo após. Cada seqüência era composta de três a sete algarismos. Nesta prova, seria também verificada a memória de lembrar coisas no futuro, a "memória de agenda". Para verificá-la, foi solicitado: "cada vez que ouvir, na seqüência de números, o número oito, terá que bater na mesa". Esta ordem deveria ser lembrada quando a prova de memória de curto prazo começasse. Trinta minutos depois, $\mathrm{AB}$ foi informada de que se iria iniciar a prova de memória de curto prazo. As instruções 
desta prova foram repetidas, mas $\mathrm{AB}$ não foi lembrada que deveria bater na mesa ao ouvir o número oito.

\section{Resultados}

$\mathrm{AB}$ apresentou fala taquilálica, na primeira entrevista. Falou bastante, sem dar muito espaço para seu interlocutor. Não mostrou dificuldades de fonologia, semântica ou sintaxe. Seus relatos eram adequados, mostrando bom raciocínio. Entretanto seu discurso era, muitas vezes, entrecortado. Freqüentemente dizia ter-se esquecido do que estava falando e perdia-se na organização de seu discurso. Ao contar sua história, relatou melhora em lembrar fatos de sua vida: AB lembrou-se perfeitamente dos fatos anteriores à sua doença, e relatou melhoras na lembrança dos fatos após seu acidente. A memória semântica foi examinada com perguntas sobre datas e fatos históricos e $\mathrm{AB}$ não apresentou problemas. No teste Mini Mental, $A B$ não apresentou nenhum problema, obtendo o escore máximo (30).

$\mathrm{Na}$ área de raciocínio, cujas tarefas podem estar eliciando mecanismos cognitivos bastante distintos, $\mathrm{AB}$ apresentou dificuldades apenas nos testes da Torre de Hanoi e de Wisconsin. No teste de Wisconsin, num total de 128 apresentações, $A B$ conseguiu realizar apenas a primeira categoria; ela atingiu o nível conceitual de $25 \%$, apresentando $24 \%$ de erros perseverativos. Fica, então, evidente que dificuldades de perceber mudanças de perspectiva de outra pessoa não estão restritas aos aspectos pragmáticos da comunicação lingüística. $\mathrm{Na}$ Torre de Hanói, suas dificuldades ocorreram quando foi preciso lidar com situações conflitantes na planificação, ou seja, quando precisava colocar a argola no pino não alvo, para manter a regra "uma argola pequena sobre uma grande".

O desempenho de $\mathrm{AB}$ nas provas de atenção, também, mostra resultados aparentemente antagônicos, se não forem pesquisados os mecanismos específicos eliciados por tarefa. $\mathrm{Na}$ prova de cancelamento, $\mathrm{AB}$ não apresentou dificuldades: cancelou nove a onze letras em cada linha, mantendo o mesmo desempenho durante a prova. Também realizou rapidamente o Trail making (terceira série: 26 segundos), mas mostrou lentidão e efeito exacerbado de interferência na Prova de Stroop (Figura 2).

Nas provas de memória, apenas as tarefas de memória prospectiva encontram-se falhas. AB teve bastante facilidade em memória de curto prazo (span de dígitos e de palavras, retendo séries de sete ítens, tanto em dígitos como em palavras). Conseguiu, com facilidade e sem erros, realizar a memória operacional com interferência em intervalos de 10 e 20 segundos. Como vimos, também sua memória semântica, verificada na conversa espontânea, estava intacta.

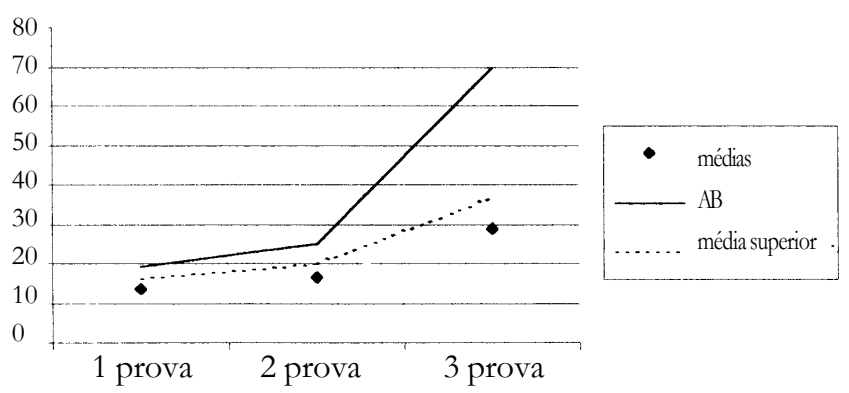

Figura 2. Resultados do desempenho de AB e normas apresentadas em Spreen e Strauss (1991) no teste de Stroop. O valor das ordenadas corresponde ao tempo medido em segundos.

Quanto à prova de Rey, a quantidade de palavras memorizadas por $\mathrm{AB}$ é maior do que as normas superiores americanas (Spreen \& Strauss, 1991), e sua curva de aprendizagem é semelhante a dos normais (Figura 3). Observa-se que foi bastante eficaz em evitar a interferência da lista B e que manteve, até o fim da sessão, as informações memorizadas. Nas duas últimas repetições, utilizou-se de estratégias de grupos semânticos, mostrando um adequado raciocínio lingüístico. Entretanto, o grande número de repetições e suas verbalizações evidenciam uma dificuldade de autoregulação. Não se lembrou do que já havia falado e a examinadora, em algumas tentativas, precisou avisá-la de que já havia falado quase todas as palavras/estímulo, o que a fez parar a evocação de palavras.

$\mathrm{AB}$ esqueceu-se de ambas tarefas de memória prospectiva. $\mathrm{Na}$ sessão seguinte, relatou que se lembrara da solicitação do recado quando estava em casa e percebera que tinha esquecido e $\mathrm{AB}$ fez o telefonema ao

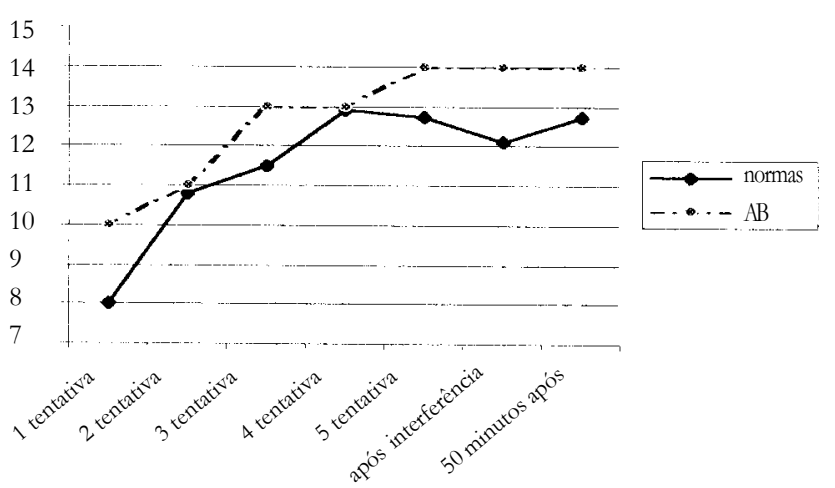

Figura 3. Teste de REY - curva de aprendizagem de AB e normas encontradas em Spreen e Strauss (1991). O valor das ordenadas corresponde ao número de palavras emitidas por $\mathrm{AB}$. 
examinador um dia depois (deslocamentos temporais). No experimento para discriminar entre o desempenho em memória imediata e o de memória prospectiva, $\mathrm{AB}$ repetiu corretamente 31 séries (88,6\% de acertos). Errou uma série entre nove, de seis dígitos e três entre quatro, de sete dígitos. Entretanto, não se lembrou da ordem para testar memória prospectiva. Tendo terminado essa repetição, $\mathrm{AB}$ foi lembrada da ordem, e repetiu a prova executando perfeitamente as duas tarefas (repetição e atenção ao número oito). Esse desempenho parece evidenciar que quando $\mathrm{AB}$ é solicitada pelo examinador para executar duas tarefas simultâneas, consegue realizálas sem dificuldades. Entretanto, se esquece da tarefa quando depende de seus próprios recursos intencionais para acessar uma ação postergada.

\section{Discussão}

Este estudo de caso mostra uma paciente que apresenta queixas de dificuldades de memória e relata uma incapacidade em organizar suas atividades diárias, após lesão pré-frontal. Também percebe que perdera a sensação da passagem do tempo, o que acentua falhas na execução de uma determinada tarefa.

Os resultados dessa extensa avaliação neuropsicológica revelaram que : (1) na triagem inicial, $\mathrm{AB}$ não apresenta dificuldades cognitivas marcantes, nem problemas de estruturas lingüísticas mas, sim, falhas discursivas, evidentes quanto ao uso de regras pragmáticas de linguagem, como avaliação de intenções do interlocutor, distúrbios atencionais ou mnemônicos; (2) na bateria de lobo frontal, cujos resultados encontram-se resumidos na Tabela 1 , $\mathrm{AB}$ mostra dificuldades apenas na Torre de Hanói e na Prova de Wisconsin, assim como lentidão na tarefa de interferência do teste de Stroop; e, (3) nas diferentes provas de memória, AR tem dificuldades restritas à memória prospectiva.

Disfunções executivas, assim como de memória relacionada a aspectos temporais, são conhecidas após lesão frontal (Fuster, 1999; Rabbitt, 1997). Entretanto, em função da especificidade de suas falhas e de várias funções cognitivas adequadas, o comportamento de $\mathrm{AB}$ não foi relacionado ao traumatismo cerebral, durante dois anos. Uma das razões desta negligência é que $\mathrm{AB}$ apresenta excelentes habilidades lingüísticas, ao se considerar as estruturas fonológica, sintático/semântica e morfológica. Ao narrar sua história, assim como em provas de raciocínio lingüístico, demonstrou boa capacidade de abstração e de formação conceitual. A preservação do raciocínio lógico e lingüístico também é evidenciada nas seguintes provas: minimental; abstração de informações

Psicologia: Reflexão e Crítica, 2001, 14(2), pp. 343-352 e de propriedades; formulação de estratégias; resolução de problemas; assim como, o recontar de uma história.

Na comunicação oral, suas dificuldades são de ordem pragmática. Fala bastante e dá pouco tempo ao interlocutor. Não apresenta confabulações, freqüentes em lesados cerebrais com danos no lobo frontal direito (Moscovitch \& Melo, 1997). Tem, entretanto, dificuldades evidentes para controlar o tempo de seu discurso e para manter a direção de suas idéias. Estas duas dificuldades evidenciam falhas no processo de supervisão (SAS Supervisory Activing System), um mecanismo mental que avalia as emissões já produzidas na elaboração de uma mensagem e controla o ponto de vista do interlocutor que recebe tal mensagem (Burgess \& Shallice, 1997).

Seu discurso é intercortado, porque o processo de supervisão (SAS) falho, não ativa nem controla a formação de novos esquemas de ações. Quando rotinas habituais ou ativadas para a realização de uma tarefa não são suficientes para finalizá-la, o SAS intervém e elicia a resposta apropriada (Parkin, 1997). Para Shallice (1988) e Normam e Shallice (1986), o sistema de supervisão - um elemento da memória prospectiva - quando falho, refletese em distúrbios da função executiva encontrados após lesão do lobo pré-frontal.

Seu desempenho no teste de Rey, diferentemente de pacientes com amnésia temporal, evidencia uma boa retenção de novas informações (Petrides, 1997). Como em caso de lesões frontais, as falhas de $\mathrm{AB}$ ocorrem em situações específicas e são detectadas em certos aspectos do desempenho (Milner e cols., 1991; Jones-Gotman e cols., 1997). Nesse sentido, somente as inúmeras respostas perseverativas de $\mathrm{AB}$ desviam-se do padrão normal. Este tipo de resposta também pode ser explicado por uma disfunção do processo de supervisão de sua emissão oral, apesar de $\mathrm{AB}$ apresentar uma metamemória preservada, por perceber falta de controle do que tinha ou não falado.

A questão que colocamos aqui é se a ausência deste processo de supervisão irá afetar também a atividade do executivo central da memória operacional (working memory), como propôs Baddeley (1986). Se a característica principal do executivo central é seu recurso atencional, $\mathrm{AB}$ teria dificuldades de realizar provas de atenção. É bastante conhecido o envolvimento do lobo frontal em processos atencionais. Richer e Lapage (1996) mostram, entretanto, em uma revisão de casos clínicos, que podem ocorrer diferentes distúrbios atencionais: dificuldades de concentração, manutenção, orientação visual e de susceptibilidade à interferência.

$\mathrm{Na}$ prova de manutenção da atenção (Prova de cancelamento de Brickenkamp) e nas de execução de duas tarefas simultâneas, $\mathrm{AB}$ teve um desempenho adequado. 
Em nenhuma prova foi observada dificuldade de concentração nem de orientação visual. No modelo de memória operacional de Baddeley (1986), o processo atencional mais importante é a atenção dividida, que, em $A B$, está intacta, visto seu desempenho na segunda etapa do estudo experimental.

A única dificuldade de $\mathrm{AB}$ nas provas de atenção foi o controle da interferência na prova de Stroop. Esta falha foi observada em pacientes com lesão frontal por Richer e Lapage (1996) em um estudo experimental que os comparou com pacientes portadores de lesão no lobo temporal. A dificuldade dos primeiros foi interpretada por uma maior susceptibilidade à interferência, dificultando as tarefas atencionais. Podemos interpretar a dificuldade de $\mathrm{AB}$ no teste Stroop em termos de maior exigência de recursos atencionais: para quem é alfabetizado, é praticamente impossível ver uma palavra escrita sem acessar seu significado, mas é extremamente fácil interromper a emissão de uma seriação solicitada no Trail Making, mesmo que neste teste seja preciso controlar duas seriações intercaladas. Outra interpretação salienta os múltiplos componentes atencionais, mostrando que a divergência desses resultados é de caráter qualitativo: o automatismo da leitura exige uma atenção interna, pois o indivíduo precisa controlá-lo para não ler em voz alta. A seriação exige, entretanto, uma atenção externa que controla letras e números dispostos no papel. Desta forma, diferentes tipos de atenção estão envolvidos nas tarefas apresentadas a AB para examinar atenção (Sevilla, 1997).

A maior susceptibilidade de $\mathrm{AB}$ à interferência pode também estar relacionada com o processo de supervisão e não com uma capacidade em realizar tarefas simultâneas ou complexas. Esse processo também pode ser o responsável pela dificuldade de tomar decisões nas compras, apesar do adequado raciocínio matemático, e pela dificuldade na prova de Wisconsin. Esta prova, apesar muito utilizada na avaliação de pacientes frontais, tem sido questionada tanto por sua sensibilidade para lesões frontais (Anderson, Damasio, Jones \& Tranel, 1991; Anderson, Bigler \& Blatter, 1995) como por sua especificidade (Bowden, 1998). Como escrevem Anderson e colaboradores (1995), o teste de Wisconsin "não pode ser interpretado de forma isolada como um índice de lesão frontal” (p. 901). Esta discussão não interfere no interesse da análise cognitiva em detectar o distúrbio básico. A incapacidade de adaptar-se às novas exigências do teste, apresentadas de forma não explícita, pode ser decorrente de um distúrbio no SAS, responsável pela formação de novos esquemas de ações (Normam \& Shallice, 1986; Parkin, 1997; Shallice, 1988).
Outra prova da bateria de lobo frontal que $A B$ apresentou muita dificuldade foi a Torre de Hanoi. Tal dificuldade evidencia falhas em processos antecipatórios, e não de coordenação de ações simultâneas, exigida pela prova de memória operacional, que $\mathrm{AB}$ realiza sem dificuldades. Estudos com PET evidenciam que a região rostral do lobo frontal é ativada diferencialmente na Torre de Londres (semelhante à Torre de Hanói), comparandose com as regiões ativadas em tarefas de memória operacional (Baker e cols., 1996). Esta atividade requer um planejamento de ações futuras bem como a manutenção desse planejamento por determinado período (Morris, Feigenbaum, Bullock \& Polkey, 1997).

AB localizava-se no espaço e no tempo, apesar de se referir à "perda da noção temporal". Este processo, de controle interno (self driven), assume dois aspectos: (1) um mecanismo de supervisão e de avaliação de suas próprias tarefas e atitudes do outro (avaliação crítica), assim como, (2) mecanismos intencionais que precisam ser autoativados mais tarde em tempos preestabelecidos (Figura 4).

Quando existe uma distância entre o planejamento da tarefa e sua execução, a dificuldade de memória de AB fica evidente. Não é uma dificuldade na recepção, no armazenamento ou na evocação de informações. Elas são estocadas e recuperadas adequadamente. Sua falha está na ativação interna não controlada pelo meio ambiente. Normalmente, um indivíduo pode dirigir suas ações por estimulação do meio ambiente ou por estimulação interna. AB consegue planejar adequadamente suas ações quando dirigidas pelo ambiente (ou por outras pessoas), mas tem muita dificuldade nas auto-ativações, principalmente quando estas precisam competir com as do meio ambiente.

A memória prospectiva é conhecida por ter uma base temporal (time-based), o que requer também ativação da

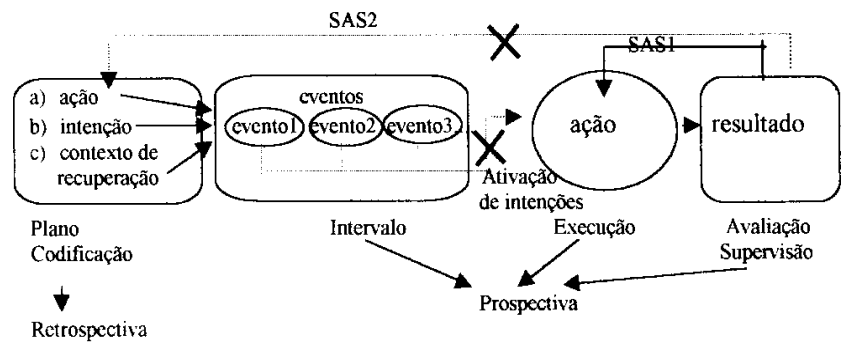

Figura 4. Rupturas na memória prospectiva em AB. As linhas pontilhadas e os X indicam rupturas na ativação de intenções e no sistema de supervisão da planificação (SAS2).

região pré-frontal (Cockburn, 1994; Rubia e cols., 1998). No modelo de memória prospectiva apresentado, 
podemos dizer que em $\mathrm{AB}$, a fase de planejamento encontra-se preservada, assim como a de execução da ação. Entretanto estão falhas, tanto a ativação de intenções a partir de eventos predeterminados, como a avaliação dos resultados da planificação (SAS2), mas não os da execução (SAS1).

\section{Conclusão}

As queixas de $\mathrm{AB}$ em relação à dificuldade de perceber o tempo, de se organizar e seus distúrbios de memória decorrem de falhas do processo de ativação de intenções após certo período de tempo, e do processo de supervisão. Podemos, então, propor que suas dificuldades específicas têm como base processos de regulação interna que prejudicam atividades relacionadas com planejamentos futuros, o que fica bastante evidente na incapacidade em realizar tarefas planejadas para um tempo postergado. Tais falhas não afetam recursos atencionais necessários na execução de ações complexas. Entretanto, são evidentes suas dificuldades em ativar intenções prévias, com falhas em mecanismos de auto-iniciação.

Este estudo evidencia, no plano da teoria neuropsicológica, uma dissociação entre memória operacional e memória prospectiva, duas funções cognitivas que podem ter correlatos fisiológicos diversos. Por outro lado, no plano clínico, ilustra as diversidades nas manifestações dos distúrbios decorrentes por lesões cerebrais frontais, tornando necessárias intervenções terapêuticas diversas e precisas. Para tal, é preciso que a avaliação neuropsicológica deixe de ser apenas sindrômica e torne-se interpretativa, baseando-se em modelos teóricos da Psicologia Cognitiva.

\section{Referências}

Ades, C. (1993). Múltiplas memórias. Psicologia USP, 4, 9-24.

Anderson, C. V., Bigler, E. D. \& Blatter, D. D. (1995). Frontal lobe lesions, diffuse damage, and neuropsychological functioning in traumatic brain-injured patients. Journal of Clinical and Experimental Neuropsychology, 17, 900-908.

Anderson, S. W., Damasio, H., Jones, E. D. \& Tranel, D. (1991). Wisconsin card sorting performance as a measure of frontal lobe damage. Journal of Clinical and Experimental Neuropsychology, 13, 909-922.

Atkinson, R. C. \& Shiffrin, R. M. (1971). The control of short-term memory. Scientific American, 225, 82-90.

Baddeley, A. D. (1986). Working memory. Oxford, Oxford: University Press. Baddeley, A. D. \& Hitsh, G. (1974). Working memory. Em G. H. Bower (Org.), The psychology of learning and motivation: Advances in research and theory (Vol. 8, pp. 47-89). New York: Academic Press.

Baker, S. C., Rogers, R. D., Owen, A. M., Frith, C. D., Dolan, R. J., Frackowiak, R. S. J. \& Robbins, T. W. (1996). Neural systems engaged by planning: A PET study of the Tower of London task. Neuropsychologia, 34, 515526.
Bowden, S. C., Fowler, K. S., Bell, R. C., Whelan, G., Clifford, C. C., Ritter, A. L. \& Long, C. M. (1998). The reliability and internal validity of the Wisconsin card sorting test. Neuropsychological Rehabilitation, 8, 243-254.

Brandimonte, M., Einstein, G. O. \& McDaniel, M. A. (1996). Prospective memory: Theory and applications. Mahwah: Lawrence Erlbaum.

Burgess, P. W. \& Shallice, T. (1997). The relationship between prospective and retrospective memory: neuropsychological evidence. Em M.A. Conway (Org.), Cognitive models of memory (pp. 247-273). Cambridge: Massachussetts.

Cadillac, C., Virbel, J. \& Nespoulous, J. L. (1995). Compréhension et mémorisation de textes de differentes structures par des sujects normaux et pathologiques: "le viel bomme”. Isbergues: L'Ortho-Edition.

Chaves, M. L. \& Izquierdo, Y. (1992). Diferential diagnosis between dementia and depression: A study of efficiency increment. Acta Neurologica Scandinavia, 85, 378-382.

Cockburn, J. (1994, Agosto). Time-based and event-based prospective memory after acquired brain damage. Poster apresentado no The third Practical Aspects of Memory Conference. University of Maryland, Maryland, USA.

Cohen, G. (1991). Memory in the real world (3 ed.). Hove: Laurence Erlbaum.

Dalla Barba, G. (1993). Prospective memory: A 'new' memory system? Em F. Boller \& J. Grafman (Orgs.), Handbook of neuropsychology . (Vol. 8). New York: Elsevier Science.

Damásio, A. (1996). O erro De descartes: Emoção, razão e cérebro bumano. São Paulo: Companhia das Letras.

Einstein, G. O. \& Daniel, M. A. (1990). Normal aging and prospective memory. Journal of Experimental Psychology: Learning, Memory and Cognitition, 16, 717-726.

Ellis, J. A. (1996) Prospective memory and the realisation of delayed intentions: a conceptual framework for research. Em M. A. Brandimonte, G. O. Einstein, M. A. McDaniel (Orgs.), Prospective memory: theory and aplications (pp. 92-104). Mahwah: Laurence Erlbaum.

Fuster, J. M. (1999). Synopsis of function and disfunction of the frontal lobe. Acta Psycbiatrica Scandinavica, 99, 51-57

Gainotti, G. (1994). Bases neurobiologiques et contrôle des émotions. Em X. Seron \& M. Jeannerod (Orgs.), Neuropsychologie bumanine (pp. 471487). Liège: Mardaga.

Goschke, T. \& Kuhl, J. (1996). Remembering what to do: explicit and implicit memory for intentions. Em M. Brandimonte, G. O. Eisntein \& M. A. McDaniel (Orgs.), Prospective memory: Theory and applications (pp. 53-91). Mahwah: Laurence Erlbaum.

Jones-Gotman M., Zatorre, R. J., Olivier, A., Andermann, F., Cendes, F., Staunton, H., McMackin, D. Siegel, A. M. \& Wieser, H-G. (1997). Learning and retention of words and designs following excision from medial or lateral temporal-lobe strutures. Neuropsychologia, 35, 963-973.

Kvavilashvili, L. (1987). Remembering intention as a distinct form of memory. British Journal of Psychology, 78, 507-518

Luria, A. R. (1966). Higher cortical functions in man. New York: Basic Books.

Mayes, A. R. \& Daum, I. (1997). How specific are the memory and other cognitive deficits caused by frontal lobe lesions? Em P. Rabbit (Org.), Methodology of frontal and executive function (pp. 471-487). Hove: Psychological Press.

Milner, B., Corst, F. \& Leonard, G. (1991). Frontal-lobe contribution to recency judgements. Neuropsychologia, 29, 601-618.

Morris, R. G., Miotto, E. C., Feigenbaum, J. D., Bullock, P. \& Polkey, C. E. (1997). The effect of goal-subgoal conflict on planning ability after frontal - and temporal-lobe lesions in humans. Neuropsychologia, 35, 1147-1157.

Moscovitch, M. \& Melo, B. (1997). Strategic retrieval and the frontal lobes: Evidence from confabulation and amnesia. Neuropsychologia, 35, 10171034.

Norman, D. A. \& Shallice, T (1986). Attention to action: willed and automatic control of behaviour. Em R.J. Davidson, G. E. Schartz \& D. Shapiro (Orgs.), Consciouness and self-regulation (pp. 1-18). New York: Plenum Press. 
Parente, M. A. M. P., Capuano, A. \& Nespoulous, J-L. (1999). Ativação de modelos mentais no recontar de histórias por idosos. Psicologia Reflexão e Crítica, 12, 157-173.

Parkin, A. (1997). Normal age-related memory loss and its relation to frontal lobe disfunction. Em P. Rabbit (Org.), Methodology of frontal and executive function (pp. 177-190). Hove: Psychological Press.

Petrides, M. (1997). Visuo-motor conditional associative learning after frontal and temporal lesions in the human brain. Neuropsychologia, 35, 989-997.

Rabbitt, P. (1997). Methodology of frontal and executive function. Hove: Psychology Press.

Richardson, J. T. (1996). Envolving concepts of working memory. Em J. Richardson, R., Engel, L. Hasher, R. Logue, E. Staltzfus \& R. Zacks (Orgs.), Working memory and buman cognition (pp. 2-30). New York: Oxford Press.

Richer, F. \& Lepage, M. (1996). Frontal lesions increase post-target interference in rapid stimulus streams. Neuropsychologia, 34, 509-514.

Rubia, K., Overmeyer, S., Taylor, E., Brammer, M., Williams, S., Simmons, A., Andrew, C. \& Bullmore, E. (1998). Prefrontal involvement in "temporal bridging" and timing movement. Neuropsychologia, 36, 12831293.

Searle, J. R. (1983). Intentionality: An essay in the philosophy of mind. Cambridge: Cambridge University Press.

Sevilla, J. G. (1997). Psicología de la atención. Madrid: Editorial Síntesis.

Shallice, N. (1988). From neuropsychology to mental structure. Cambridge: Cambridge University Press.

Spreen, O. \& Strauss, E. (1991). A compendium of neuropsychological tests. New York: Oxford University Press.
Stuss, D. T. \& Benson, D. F. (1986). The frontal lobes. New York: Raven Press. Van Der Linden, M. (1994). Neuropsychologie de la memoire. Em X. Seron \& M. Jeannerod (Org.), Neuropsychologie bumanine (pp. 282-316). Liège: Mardaga.

Xavier, G. F. (1993). A modularidade da memória e o sistema nervoso. Psicologia USP, 4, 61-115.

Xavier, G. F. (1996). Memória: correlatos anatomo-funcionais. Em R. Nitrini, P. Caramelli \& L. L. Mansur (Orgs.), Neuropsicologia, das bases anatômicas à reabilitação (pp. 107-126). São Paulo: Clínica Neurológica Hospital de Clínicas FMUSP.

Wheeler, M. A., Stuss, D. T. \& Tulving, E. (1997). Toward a theory of episodic memory: The frontal lobes and autonoetic consciousness. Psychological Bulletin, 121, 331-354

Wiggs, C. L., Weiseberg, J. \& Martin, A. (1999). Neural correlates of semantic and episodic memory retrieval. Neuropsychologia, 37, 103-118.

Wilkins, A. J. \& Baddeley, A. D. (1978). Remember to recall in everyday life: An approach to absent-mindedness. Em M. M. Gruneberg, P. E. Moris \& R. N. Sykes (Orgs.), Practical aspects of memory: Current research and issues (pp. 29-48). London: Academic Press.

Sobre os autores

Maria Alice de Mattos Pimenta Parente é Doutora em Psicologia pela Universidade de São Paulo, Membro da Assembléia de Pesquisadores do Centro de Pesquisas do Centro Hospitalar Côte-desNeiges da Universidade de Montreal Canadá, Professora do Departamento de Psicologia do Desenvolvimento e da Personalidade da Universidade Federal do Rio Grande do Sul, Membro da Junta diretora da Sociedade Latino Americana de Neuropsicologia.

Mônica Sparta é Psicóloga, Mestranda do Curso de Pós-Graduação em Psicologia do Desenvolvimento UFRGS.

André Luiz Palmini é Neurologista, Professor da Faculdade de Medicina da Pontifícia Universidade Católica do Rio Grande do Sul. 